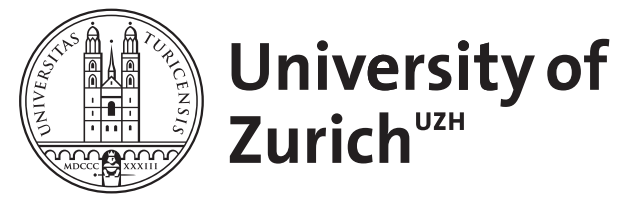

\title{
Endocardial irrigated catheter for volumetric optoacoustic mapping of radio-frequency ablation
}

\author{
Özsoy, Çağla ; Deán-Ben, Xosé Luís ; Razansky, Daniel
}

DOI: https://doi.org/10.1117/12.2527167

Posted at the Zurich Open Repository and Archive, University of Zurich ZORA URL: https://doi.org/10.5167/uzh-198760

Conference or Workshop Item

Published Version

Originally published at:

Özsoy, Çă̆la; Deán-Ben, Xosé Luís; Razansky, Daniel (2019). Endocardial irrigated catheter for volumetric optoacoustic mapping of radio-frequency ablation. In: European Conferences on Biomedical Optics, Munich, 2019. SPIE, 33.

DOI: https://doi.org/10.1117/12.2527167 


\section{Endocardial irrigated catheter for volumetric optoacoustic mapping of radio-frequency ablation}

Özsoy, Çağla, Deán-Ben, Xosé Luis, Razansky, Daniel

Çağla Özsoy, Xosé Luis Deán-Ben, Daniel Razansky, "Endocardial irrigated catheter for volumetric optoacoustic mapping of radio-frequency ablation," Proc. SPIE 11079, Medical Laser Applications and Laser-Tissue Interactions IX, 110790X (22 July 2019); doi: 10.1117/12.2527167

SPIE. Event: European Conferences on Biomedical Optics, 2019, Munich, Germany 


\title{
Endocardial Irrigated Catheter for Volumetric Optoacoustic Mapping of Radio-Frequency Ablation
}

\author{
Çağla Özsoy ${ }^{1,2}$, Xosé Luís Deán-Ben ${ }^{1,2}$, and Daniel Razansky1,2,* \\ ${ }^{1}$ Faculty of Medicine and Institute of Pharmacology and Toxicology, University of Zurich, \\ Switzerland \\ ${ }_{2}^{2}$ Institute for Biomedical Engineering and Department of Information Technology and Electrical \\ Engineering, ETH Zurich, Switzerland \\ *Corresponding author:daniel.razansky@uzh.ch
}

\begin{abstract}
Radio-frequency (RF) catheter ablation is routinely used in the clinics to treat arrhythmias, neoplastic lesions and other dysfunctional tissues. It is based on scarring the target tissue via localized heating induced by medium frequency alternating current in the proximity of a metallic ablation catheter. The outcome of RF interventions strongly relies on the temperature distribution within the treated tissue as well as on the exposure time, which cannot be efficiently monitored with existing clinical tools. Optoacoustic (OA) tomography has recently been shown to provide unique capabilities for RF ablation monitoring such as high spatio-temporal resolution, hand-held operation feasibility and high sensitivity to temperature changes and tissue coagulation. However, shallow light penetration in biological tissues strongly limits the accessible regions, particularly for cardiac procedures, while internal illumination through the catheter induces strong responses in the metallic parts that hamper soft tissue visualization. Herein, we present a new endocardial catheter based on saline irrigation that delivers electric current while being transparent for light. The new design averts OA image artifacts due to the presence of metallic electrodes while retaining the ablation efficiency.
\end{abstract}

Keywords: Optoacoustic, photoacoustic, rf ablation, catheter, temperature

\section{INTRODUCTION}

Radiofrequency (RF) catheter ablation is commonly used in numerous clinical interventions including cancer therapy, endovenous removal of varicose veins or treatment of cardiac rhythm disorders [1]. RF ablation is generally preferred over alternative thermal ablation approaches due to its low cost, minimal invasiveness and simplicity of operation. The success rates of RF catheter ablation procedures critically depend upon the electrical impedance of the treated area, which is related to the thermal-induced tissue alteration. Availability of non-invasive monitoring tools capable of assessing lesion progression in real time is hence essential for optimizing the treatment outcomes $[2,3]$.

Recently, optoacoustic (OA) tomography has been proposed as a novel and advantageous approach for two- and threedimensional mapping of the temperature distribution and lesion progression during RF ablation procedures [4-7]. The OA effect is based upon excitation of ultrasound waves by short light pulses and is highly sensitive to temperature changes and chemical transformations associated to tissue coagulation. These features, combined with other important advantages of OA such as high spatio-temporal resolution [8] or hand-held operation feasibility [9,10], can be exploited for efficient monitoring of thermal therapeutic interventions [11-13]. Yet, light delivery to the target tissue must be optimized in order to minimize generation of artefactual signals by metallic parts conducting the ablation current [14]. In this work, we address this challenge by designing a new endocardial ablation catheter. The catheter is based on irrigation of a conductive saline solution that is transparent to light, thus efficiently guides both electric current and OA excitation laser pulses.

Medical Laser Applications and Laser-Tissue Interactions IX, edited by Lothar D. Lilge, Carsten M. Philipp, Proc. of SPIE-OSA Vol. 11079, 110790X · C 2019 SPIE-OSA · CCC code: 1605-7422/19/\$21 · doi: 10.1117/12.2527167 


\section{MATERIALS AND METHODS}

\subsection{Ablation catheter}

Figure 1 shows a schematic description of the newly introduced RF ablation catheter. Electrical conductivity is provided via irrigation of a saline solution that avoids direct contact between the tissue and metallic electrodes. The catheter tip further encapsulates a custom fiber delivery system for OA signal excitation. In addition, a metallic needle acts as an ablation electrode and facilitates saline irrigation. A T-type thermocouple is further attached to the catheter for temperature measurement. By positioning the metallic electrode several millimeters away from the catheter tip, no OA image artifacts are produced by light absorption at the fiber tips or acoustic reflections by the metallic catheter end.

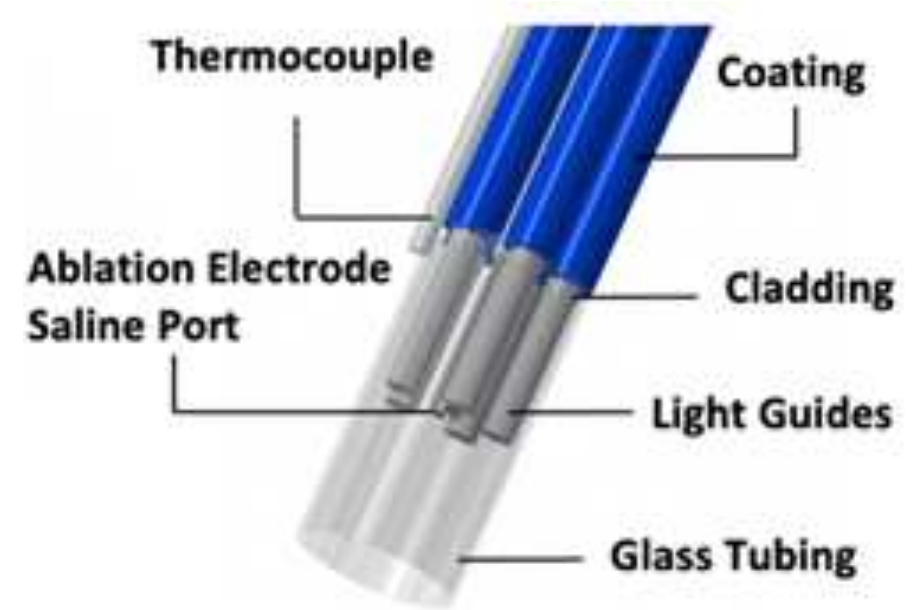

Figure 1. Schematics of the newly introduced endocardial irrigated catheter.

\subsection{Three-dimensional optoacoustic imaging system}

The ultrasound waves optoacoustically generated by light excitation through the catheter can be detected at several points outside the body. In this work, volumetric OA imaging was performed with a 256-element spherical transducer array with $4 \mathrm{MHz}$ central frequency and $100 \%$ detection bandwidth, where all signals were simultaneously acquired at 40 megasamples per seconds. A detailed description of the OA imaging system and tomographic reconstruction procedure can be found elsewhere [15].

\subsection{Cardiac ablation experiments}

The performance of the suggested approach was tested by monitoring the RF ablation of a porcine heart at several locations ex-vivo. The heart chambers were filled with blood to mimic the in vivo conditions. Both ventricle and atrium were ablated by using an RF generator with an average power of $10 \mathrm{~W}$. 3.6\% (hypertonic) saline solution was irrigated throughout the ablation procedure at $1 \mathrm{ml} / \mathrm{min}$ rate. The laser wavelength was set to $780 \mathrm{~nm}$ corresponding to the maximum OA contrast achieved between coagulated and non-coagulated tissues [11]. OA signals were acquired for $400 \mathrm{~s}$, including $20 \mathrm{~s}$ before the RF generator was activated, $300 \mathrm{~s}$ of ablation procedure and $80 \mathrm{~s}$ of cool-down phase.

\section{RESULTS}

Figure 2 presents the experimental results obtained in the OA monitoring experiments of RF ablation of the porcine tissue. Throughout the experiments, it was observed that larger and deeper lesions were produced on the smooth wall of the ventricle, whereas the rough surface of the atrium impaired electric contact. A clear increase in the relative OA signal was perceived in the entire ROI with respect to the signal intensity level before the activation of the RF generator. This is 
mainly due to the change in Grüneisen parameter, which is related to the increase in temperature due to the resistive heating of the heart muscle [16]. Also, the OA signal after the cool- down period was higher than in the beginning, which corroborates the fact that the OA signal increased due to protein denaturation and tissue coagulation.
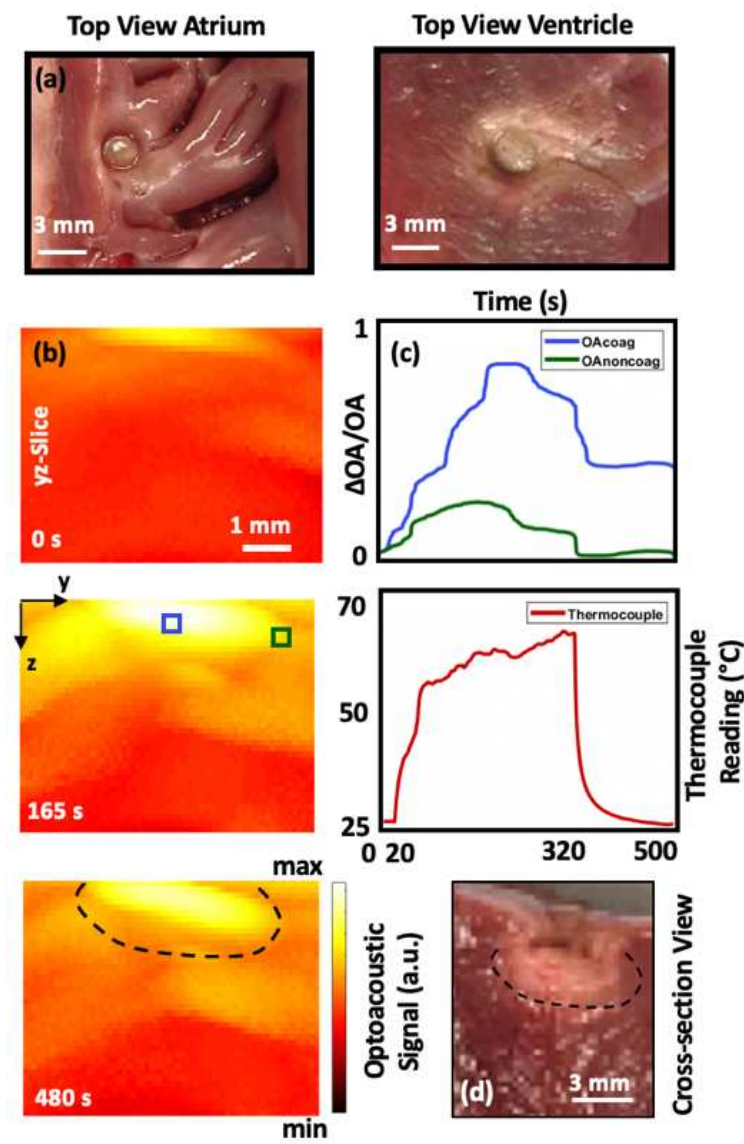

Figure 2. Real-time OA monitoring of RF ablation in excised porcine heart (a) Top view of the RF ablation lesions created in the atrium (left) and ventricle (right) (b) Lateral views of fluence corrected OA images acquired from the ventricle at different time points during the treatment; before ablation started (top), during lesion formation (middle) and a point during the cooldown period (bottom) (c) Relative change of OA signal in time for the two points in (b) (top) and thermocouple reading (bottom). (d) Cross-section of the ablation lesion created on ventricle.

\section{CONCLUSIONS}

The demonstrated feasibility of real-time OA monitoring of the lesion development during RF ablation with a salineirrigation-coupled catheter can significantly enhance effectiveness of this procedure. By avoiding direct contact of the metallic electrodes with the tissue, artefacts associated with light absorption or acoustic reflections by the electrodes can be minimized. Active cooling provided by irrigated catheters is also an established method implemented in clinical cardiac $\mathrm{RF}$ ablation procedures. This has been shown to increase the efficiency of the lesion development as well as to reduce the chances of tissue charring and the risk of steam pops. The results presented in the ex-vivo porcine heart ablation experiments demonstrate that the newly developed concept can be practically integrated in a realistic clinical scenario. Different chambers of porcine heart were successfully ablated providing uniform coagulum without any tissue charring, while the OA signal changes associated with temperature increase and chemical transformations during coagulation could be monitored in real time. 


\section{REFERENCES}

[1] Chu, K. F. and Dupuy, D. E., "Thermal ablation of tumours: biological mechanisms and advances in therapy," Nat Rev Cancer. 14(3), 199-208 (2014).

[2] Ni, Y., Mulier, S., Miao, Y., Michel, L. and Marchal, G., "A review of the general aspects of radiofrequency ablation," Abdom Imaging 30(4), 381-400 (2005).

[3] Tsoumakidou, G., Koch, G., Caudrelier, J., Garnon, J., Cazzato, R. L., Edalat, F. and Gangi, A., "Image-guided spinal ablation: a review," Cardiovasc Intervent Radiol 39(9), 1229-1238 (2016).

[4] Meng, L., Deschaume, O., Larbanoix, L., Fron, E., Bartic, C., Laurent, S., Van der Auweraer, M. and Glorieux, C., "Photoacoustic temperature imaging based on multi-wavelength excitation," Photoacoustics 13, 33-45 (2019).

[5] Petrova, E. V., Brecht, H. P., Motamedi, M., Oraevsky, A. A. and Ermilov, S. A., "In vivo optoacoustic temperature imaging for image-guided cryotherapy of prostate cancer," Phys. Med. Biol. 63(6), 064002 (2018).

[6] Landa, F. J. O., Deán-Ben, X. L., Sroka, R. and Razansky, D., "Volumetric optoacoustic temperature mapping in photothermal therapy," Scientific Reports 7(1), 9695 (2017).

[7] Landa, F. J. O., Penacoba, S., Montero de Espinosa, F., Razansky, D. and Deán-Ben, X. L., "Four-dimensional optoacoustic monitoring of tissue heating with medium intensity focused ultrasound," Ultrasonics 94, 117-123 (2019).

[8] Deán-Ben, X. L., Fehm, T. F., Ford, S. J., Gottschalk, S. and Razansky, D., "Spiral volumetric optoacoustic tomography visualizes multi-scale dynamics in mice," Light: Science \& Applications 6(4), e16247 (2017).

[9] Deán-Ben, X. L., Fehm, T. F. and Razansky, D., "Universal hand-held three-dimensional optoacoustic imaging probe for deep tissue human angiography and functional preclinical studies in real time," J Vis Exp. 93, 51864 (2014).

[10] Schellenberg, M. W. and Hunt H. K., "Hand-held optoacoustic imaging: A review," Photoacoustics 11, 1427 (2018).

[11] Pang, G. A., Bay, E., Deán-Ben, X. L. and Razansky, D., "Three-dimensional optoacoustic monitoring of lesion formation in real time during radiofrequency catheter ablation," Journal of Cardiovascular Electrophysiology 26(3), 339-345 (2015).

[12] Fehm, T. F., Deán-Ben, X. L., Schaur, P., Sroka, R. and Razansky D., "Volumetric optoacoustic imaging feedback during endovenous laser therapy-an ex vivo investigation," Journal of Biophotonics 9(9), 934-941 (2016).

[13] Iskander-Rizk, S., Kruizinga, P., van der Steen, A. F. W. and Van Soest G., "Spectroscopic photoacoustic imaging of radiofrequency ablation in the left atrium," Biomed. Opt. Express 9(3), 1309-1322 (2018).

[14] Rebling, J., Landa, F. J. O., Deán-Ben, X. L., Douplik, A. and Razansky D., "Integrated catheter for simultaneous radio frequency ablation and optoacoustic monitoring of lesion progression," Optics Letters 43(8), 1886-1889 (2018).

[15] Deán-Ben, X. L. and Razansky, D., "Portable spherical array probe for volumetric real-time optoacoustic imaging at centimeter-scale depths," Opt. Express 21(23), 28062-28071 (2013).

[16] Larina, I. V., Larin, K. V. and Esenaliev R. O., "Real-time optoacoustic monitoring of temperature in tissues," J. Phys. D: Appl. Phys. 38(15), 2633-2639 (2005). 